\title{
EUV ablation of organic polymers at a high fluence
}

\author{
Chiara Liberatore ${ }^{1,2}$, Klaus Mann $^{3}$, Matthias Müller ${ }^{3}$, Ladislav Pina ${ }^{2}$, Libor Juha ${ }^{1}$, Jorge J. Rocca ${ }^{4}$, \\ Akira Endo ${ }^{1}$, and Tomas Mocek ${ }^{1}$ \\ ${ }^{1}$ HiLASE Centre, Institute of Physics ASCR, v.v.i., Za Radnicí 825, 25241 Dolní Břežany, Czech Republic \\ ${ }^{2}$ Czech Technical University, Thákurova 2077/7, 16000 Prague 6, Czech Republic \\ ${ }^{3}$ Laser Laboratorium Göttingen (LLG), 37077 Göttingen, Germany \\ ${ }^{4}$ Department of Electrical and Computer Engineering, Colorado State University, Fort Collins, CO 80523, USA \\ (Received 3 March 2014; revised 9 May 2014; accepted 3 July 2014)
}

\begin{abstract}
A preliminary investigation on short-wavelength ablation mechanisms of poly(methyl methacrylate) (PMMA) and poly (1,4-phenylene ether ether-sulfone) (PPEES) by extreme ultraviolet (EUV) radiation at $13.5 \mathrm{~nm}$ using a table-top laserproduced plasma from a gas-puff target at LLG (Göttingen) and at $46.9 \mathrm{~nm}$ by a $10 \mathrm{~Hz}$ desktop capillary discharge laser operated at the Institute of Physics (Prague) is presented. Ablation of polymer materials is initiated by photoinduced polymer chain scissions. The ablation occurs due to the formation of volatile products by the EUV radiolysis removed as an ablation plume from the irradiated material into the vacuum. In general, cross-linking of polymer molecules can compete with the chain decomposition. Both processes may influence the efficiency and quality of micro(nano)structuring in polymer materials. Wavelength is a critical parameter to be taken into account when an EUV ablation process occurs, because different wavelengths result in different energy densities in the near-surface region of the polymer exposed to nanosecond pulses of intense EUV radiation.
\end{abstract}

Keywords: EUV ablation; organic polymer; photo-erosion mechanisms; wavelength effect

\section{Introduction}

Laser ablation is an efficient removal of a material from its surface and near-surface region irradiated by an intense laser beam at a fluence above the single-shot ablation threshold ${ }^{[1,2]}$. Due to its potential use in surface patterning/structuring and other applications (e.g., pulsed laser deposition (PLD)), ablation has attracted attention from the advent of laser technology in the sixties. Among the lasers widely utilized to initiate material ablation, ultraviolet (UV) lasers are often applied because of their limited penetration depth and ability to induce photochemical ablation while avoiding thermal artefacts ${ }^{[3]}$. It is known that photochemical, non-thermal decomposition plays an important role when the wavelengths decrease. Some ablation studies at shorter wavelengths in the vacuum UV (VUV) region have been conducted, for example, with $\mathrm{F}_{2}$ excimer lasers $\left(\lambda=157 \mathrm{~nm}^{[4]}\right)$ and radiation generated by four-wave-sum-frequency mixing of a frequency-doubled Nd:YAG laser $\left(\lambda=125 \mathrm{~nm}^{[5]}\right)$. Laboratory-scaled laser sources operating at even shorter wavelengths have recently

Correspondence to: C. Liberatore, HiLASE Centre, Institute of Physics ASCR, v.v.i., Za Radnicí 825, 25241 Dolní Břežany, Czech Republic. Email: liberatore@fzu.cz become operational in the extreme UV (EUV) spectral range $(\lambda<100 \mathrm{~nm})^{[6-8]}$.

The EUV sources utilized to induce material erosion emit at both low peak power and high peak power. In principle, this results in different modes of material removal ${ }^{[9,10]}$. With low-peak-power sources, materials are removed by photo-induced desorption of their volatile fragments from the irradiated sample surface. Each EUV photon carries enough energy to break any chemical bond. This energy is also usually higher than the cohesive energy of any solids. Therefore, the energetic photons absorbed by the photoeffect in atoms located in a near-surface region may create small fragments of a sample material, which are desorbed into the vacuum.

Quite a different situation is expected if a high-peak-power source delivers a single high-energy pulse to the material. The sample is then exposed to a high local dose of radiation (given by the energy content of the pulse and the absorption length of the radiation in the irradiated material) in a short period of time (given by the pulse duration), i.e., a very high dose rate. This means that a large number of events that cause radiation-induced structural decomposition (i.e., organic polymer chain scissions, etc.) occur almost simultaneously in a relatively thick layer of irradiated material. 


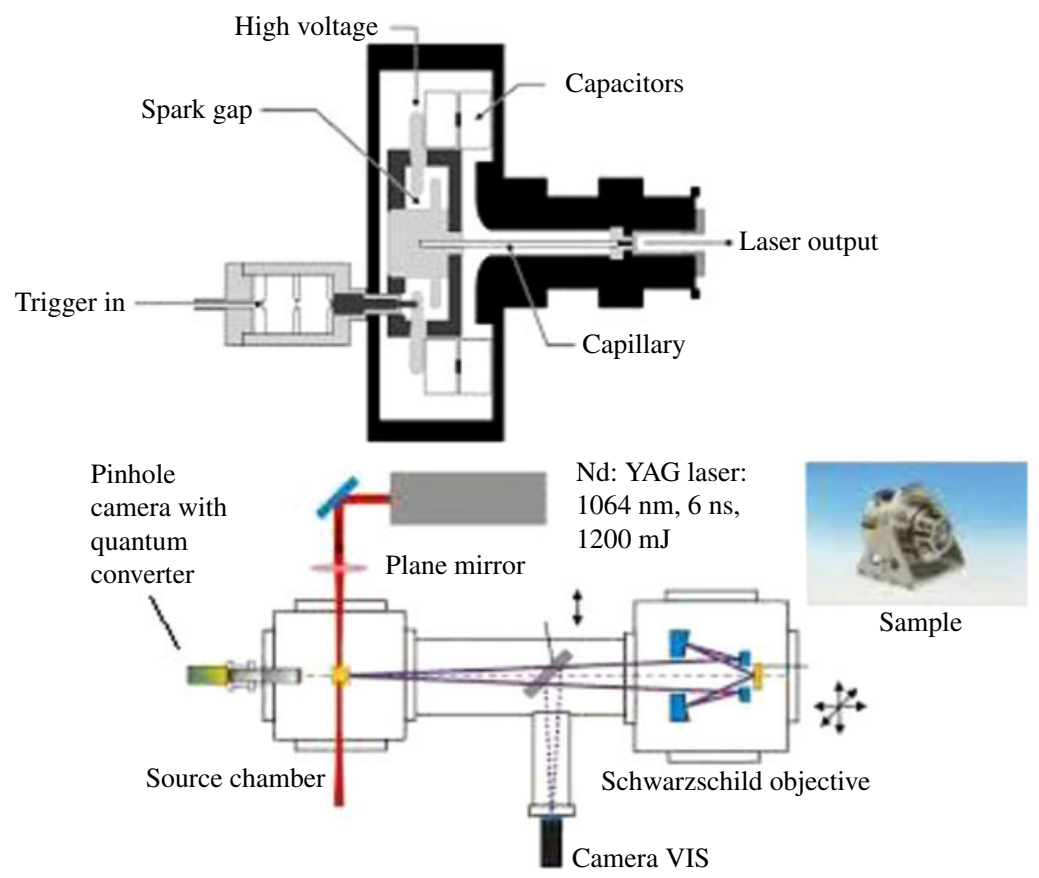

Figure 1. Experimental systems: scheme of the CDL system operated in Prague ${ }^{[15,16]}$ and scheme of the LPP system operated at LLG in Göttingen ${ }^{[17]}$.

Since a portion of the radiation energy absorbed in the material will be thermalized, the sudden heating of the layer, which is also heavily chemically altered by the radiation, must be taken into account when the fluence is above the single-shot ablation threshold. The overheated, fragmented region of the irradiated material represents a new phase, which tends to blow off into the vacuum. These particular processes, as well as specific features of short-wavelength ablation with respect to ablation induced by conventional, long-wavelength (UV-Vis-IR) sources, together with their potential applications in micro(nano)structuring, represent a main goal of our research program.

In this contribution, a comparison of ablation results obtained in poly(1,4-phenylene ether ether-sulfone) PPEES at $13.5 \mathrm{~nm}$ and $46.9 \mathrm{~nm}$ is presented.

\section{Materials and methods}

The ablation experiment has been performed at LLG using poly(methyl methacrylate) (PMMA) as a reference material and then, with the same setup, on a sample material, i.e., PPEES. The material chosen as the target to be tested is PPEES $^{[11]}$ because poly(olefin sulfone)s (POSs) are wellknown radiation sensitive polymers ${ }^{[12-14]}$. Among various POS materials, PPEES was selected because its molecule contains $\pi$ electrons, in contrast to the aliphatic molecules of PMMA.
The experimental system used in the EUV irradiation experiments is shown in Figure 1. The capillary-discharge Ne-like Ar EUV laser (CDL) ${ }^{[15]}$ developed and built at CSU in Fort Collins, installed and operated in Prague, was used to ablate PPEES by intense EUV radiation. Its output characteristics are as follows: wavelength $46.9 \mathrm{~nm}$, pulse length $1.5 \mathrm{~ns}$ (FWHM), maximum pulse energy approximately $10 \mu \mathrm{J}$, repetition rate $5 \mathrm{~Hz}$ (typical) and $12 \mathrm{~Hz}$ (maximum), capillary lifetime $(2-3) \times 10^{4}$ pulses, current $\sim 21 \mathrm{kA}$. The capillary is an $\mathrm{Al}_{2} \mathrm{O}_{3}$ (inner diameter: $3.2 \mathrm{~mm}$, length: $210 \mathrm{~mm}$ ), Ar-filled (50 Pa). A spherical multilayer $\mathrm{Sc} / \mathrm{Si}$ mirror with a focal length of $0.25 \mathrm{~m}$ was used to focus the CDL beam. The mirror was designed and manufactured to have a maximum reflectivity of $46.9 \mathrm{~nm}$ radiation for a $6^{\circ}$ angle between the incident and reflected beams ${ }^{[16]}$.

The second experimental system, built and operated at LLG, is based on a driving Nd:YAG laser (wavelength $1064 \mathrm{~nm}$, pulse length $7 \mathrm{~ns}$, jitter $<1 \mathrm{~ns}$, divergence $<0.5$ $\mathrm{mrad}-$ Gaussian profile at far field, pulse energy stability $<1 \%$ for $90 \%$ of pulses, maximum pulse energy $1.2 \mathrm{~J}$, i.e., twice that used by Barkusky et al. in earlier EUV ablation experiments ${ }^{[17]}$ ) focused into a Xe gas-puff target. EUV emission of the laser-produced Xe plasma is collected and focused by a Schwarzschild objective with a $\mathrm{Mo} / \mathrm{Si}$ multilayer coating. The source provides EUV surface energy densities (i.e., fluences) exceeding $0.1 \mathrm{~J} \mathrm{~cm}^{-2}$ at $13.5 \mathrm{~nm}$, $2 \%$ bw. 
PMMA: number of shots / ablation depth

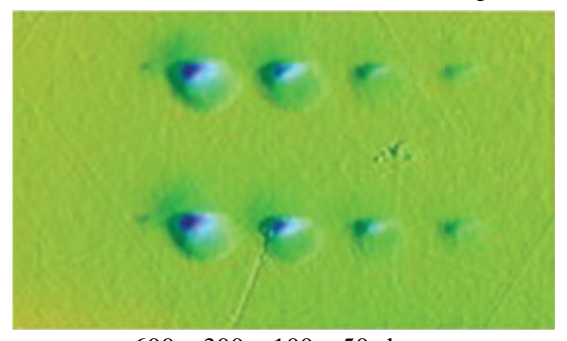

$600-300-100-50$ shots

Caustic measurement on PMMA

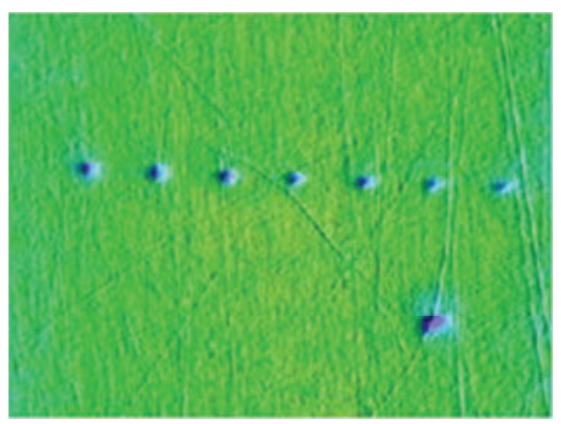

z (cm) (ablation depth)

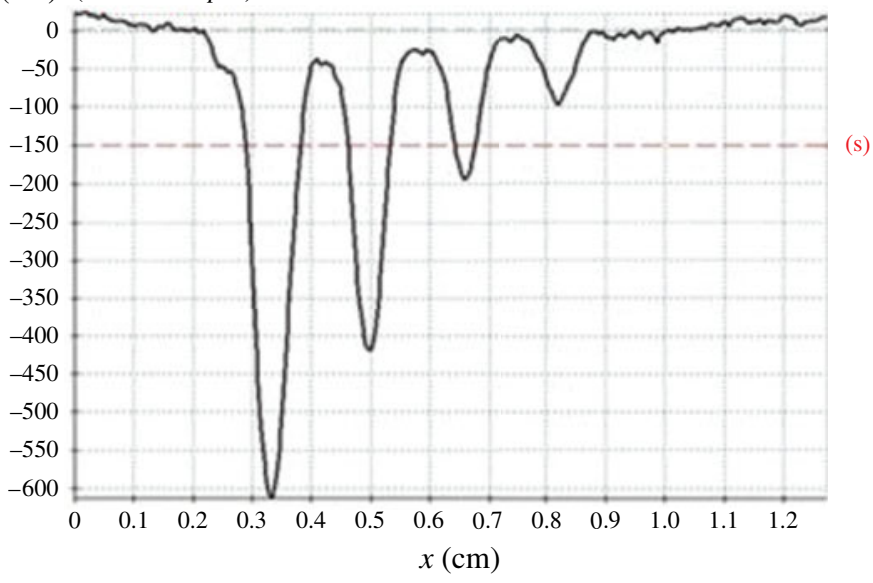

position on the target (at the focus)

$z(\mathrm{~cm})$ (ablation depth)

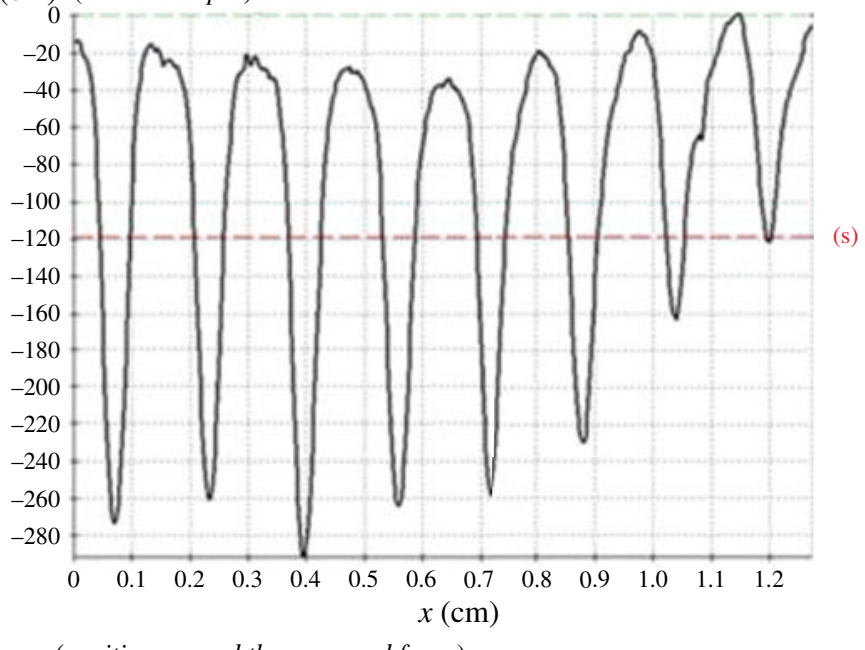

(position around the supposed focus)

Figure 2. LLG source at a wavelength of $13.5 \mathrm{~nm}$ : example of caustic (bottom) and dependence of ablation depth on the number of shots (top). Measurements on PMMA.

This second wavelength is particularly promising because it allows one to modify smaller areas of the material, which is an advantage for many applications (i.e., lithography, nanostructuring).

Typical ablation tests were performed in the tight focus, varying the number of shots. Procedures making it possible to find the focus (i.e., a measurement of caustics, $z$ scan) to determine the EUV energy distribution in the focus and to obtain other irradiation characteristics have been explained elsewhere ${ }^{[18]}$.

The caustics (Figures 2 and 3) are lines of ablated spots, obtained by moving the target in fixed steps of a few microns around the supposed best focal position. At each new position the target was irradiated by a greater number of laser shots. This procedure allows one to verify the focal position of the beam on the sample and, at the same time, to demonstrate that the polymer under test can be ablated by EUV radiation.

\section{Results}

PMMA was efficiently ablated by $13.5 \mathrm{~nm}$ radiation ${ }^{[18]}$, as shown in Figure 2. The ablation process is very stable and the craters maintain the same morphology with an increasing number of shots. Under very similar irradiation conditions, the measurement has been repeated with the PPEES sample. Surprisingly, the ablation pattern has not been observed.

This can be explained with respect to our previous positive PPEES ablation results obtained at $46.9 \mathrm{~nm}$ (Figures 3 and 4$)^{[18]}$ by recalling that the absorption of EUV photons on polymers is expressed by Lambert's law:

$$
\frac{\mathrm{d} I(z)}{\mathrm{d} z}=-\alpha I(z),
$$

where $I$ is the intensity, $z$ is the depth below the surface, and $\alpha$ is the absorption coefficient of the radiation in the material. The attenuation length is then $1 / \alpha$. For PPEES (density: 


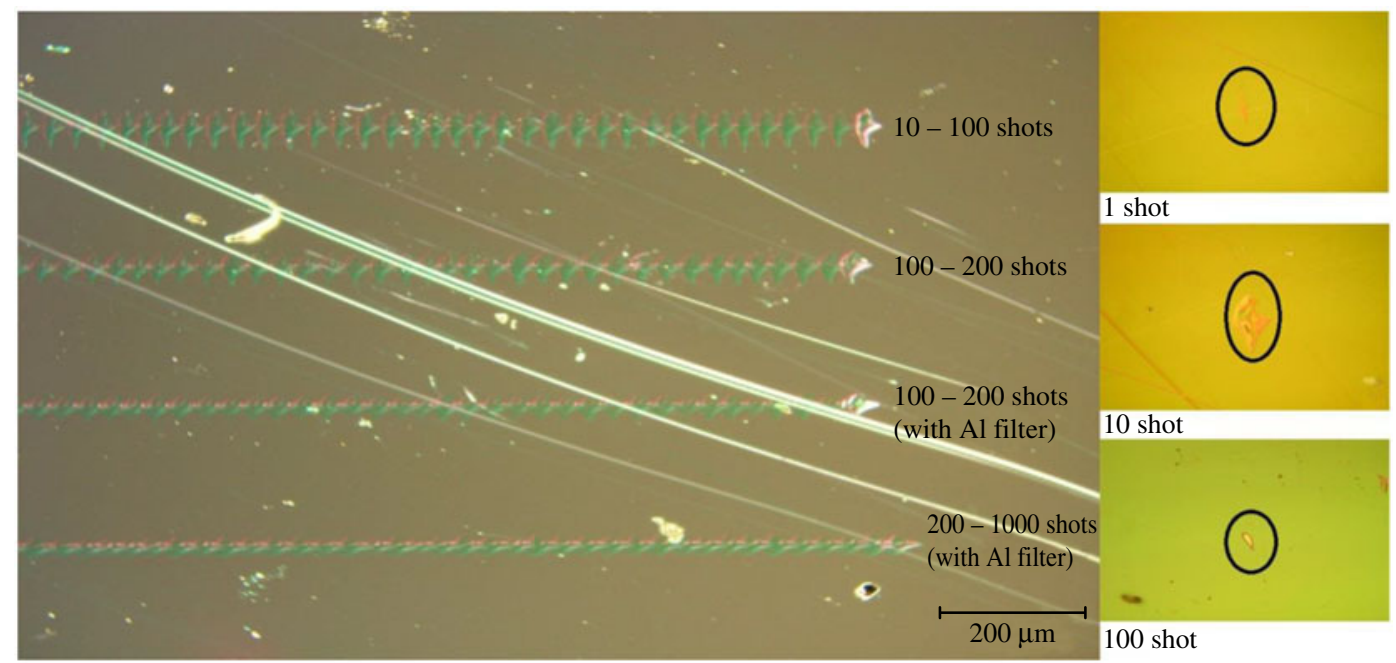

Figure 3. CDL at a wavelength of $46.9 \mathrm{~nm}$ : caustic measurement wavelength $46.9 \mathrm{~nm}$ (below) and ablation measurement (top). The shape of the crater is almost the same along the line of the caustic. Looking at the image on the right it can be seen that the annular form is preserved although, on increasing the number of shots, its dimensions increase.
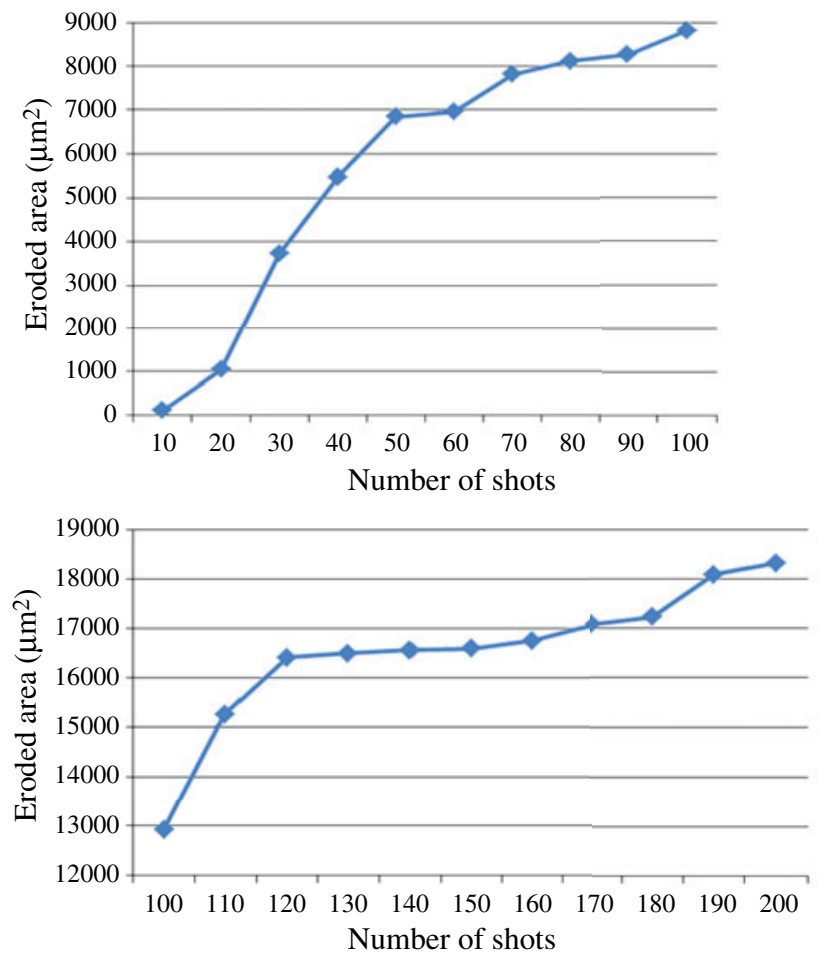

Figure 4. CDL at a wavelength of $46.9 \mathrm{~nm}$ : eroded area on number of shots (100-200 shots, bottom; 10-100 shots, top).

$1.24 \mathrm{~g} \mathrm{~cm}^{-3}$; elemental composition: $\left.\mathrm{C} 18 \mathrm{SO} 4 \mathrm{H} 12\right)$, the attenuation length at a wavelength of $13.5 \mathrm{~nm}$ (photon energy: $91.8 \mathrm{eV}$ ) is $215 \mathrm{~nm}^{[19]}$. The attenuation length at a wavelength of $46.9 \mathrm{~nm}$ (photon energy: $26.4 \mathrm{eV}$ ) is much shorter, i.e., approximately $20 \mathrm{~nm}^{[19]}$.

Therefore, there is at least one order of magnitude higher energy density in the PPEES near-surface region when irradiated at $46.9 \mathrm{~nm}$ than in the case of irradiation of the material at $13.5 \mathrm{~nm}$ at the same surface energy density. Two conclusions follow from this fact:

- at $46.9 \mathrm{~nm}$ the ablation threshold should be much lower than at $13.5 \mathrm{~nm}$;

- during the exposure at $13.5 \mathrm{~nm}$, the near-surface region is not so 'overexposed/overheated' as in the previous case (i.e., at $46.9 \mathrm{~nm}$ ), so that single-photon radiolytical processes would play an important role in material ablation, making visible a difference in the radiation stabilities of PPEES and PMMA. PPEES is much more radiation stable than PMMA because of the $\pi$ electrons in the PPEES molecules.

The differences in the efficiency of the process explained thanks to the differences in the ablation thresholds and in the efficiency of the radiolytic process mentioned above explain why no ablation was registered at $13.5 \mathrm{~nm}$ for fluences achieving a relatively high value, i.e., $127 \mathrm{~mJ} \mathrm{~cm}^{-2}$. It also makes reasonable the finding of ablation craters on the PPEES surface illuminated by $46.9 \mathrm{~nm}$ at a lower fluence, i.e., around $50 \mathrm{~mJ} \mathrm{~cm}^{-2}$.

These considerations provide evidence of the importance of the chemical structure of the material. Indeed, considering just the scaling law related to the optical systems used in this experimental campaign, results at a lower fluence would have been expected. Indeed, the resolution $\sigma$ is proportional to the wavelength:

$$
\sigma \propto \frac{\lambda}{\mathrm{NA}}
$$

where NA is the numerical aperture of the optics; while for the penetration depth:

$$
d \propto \frac{1}{\lambda}
$$


Considering the parameters of the LLG experimental system $\left(\mathrm{NA}=0,4 ; E>0.1 \mathrm{~J} \mathrm{~cm}^{-2}\right.$ and $\lambda=13.5 \mathrm{~nm}$ ) from the relation:

$$
\text { fluence } \propto \frac{E}{\sigma^{2}},
$$

a fluence of $87 \mathrm{~mJ} \mathrm{~cm}^{-2}$ must have been sufficient to ablate. Indeed, this is the case for the less radiation resistant $\mathrm{PMMA}^{[17]}$. The difference in the study between PMMA and PPEES ablation using the EUV wavelength, in particular when it is shorter, comes directly from the impossibility of using the current scaling law for the second polymer, because it is no longer valid, being affected by the chemical structure of the polymer.

Repeating the experiment on PPEES, which presents a higher radiation stability, is interesting because use of a better resistor would provide an improvement in the industrial application of EUV ablation processes. It guarantees a longer lifetime of such systems before damage.

For this reason, in the next phase of the project, we are going to increase the output energy of the LLG source and focus its EUV emission onto a smaller spot to exceed the EUV ablation threshold of PPEES at $13.5 \mathrm{~nm}$ and to evaluate the required fluence experimentally.

However, in future industrial EUV polymer ablation, the wavelength-dependent ablation depth alpha is the guiding parameter; therefore multiple EUV wavelengths may be required, necessitating the use of different elements (gas or solid).

\section{Conclusions}

In this contribution, we have demonstrated the possibility of creating well-developed ablation craters in PPEES exposed to focused $46.9 \mathrm{~nm}$ CDL radiation. However, the more penetrating $13.5 \mathrm{~nm}$ radiation (its attenuation length in PPEES exceeds $0.2 \mu \mathrm{m}$ ) was not able to ablate PPEES, although PMMA was ablated quite effectively under these irradiation conditions. The higher radiation stability of PPEES is expected because of the presence of $\pi$ electrons in the PPEES molecule, in contrast to the less radiation resistant polymer structures (PMMA). The increased attenuation length at the shorter wavelength is likely responsible for the observed difference in the response of PPEES to radiation emitted by the CDL source $(\lambda=46.9 \mathrm{~nm})$ and the LLG source $(\lambda=13.5 \mathrm{~nm})$.

\section{Acknowledgements}

This work benefitted from: the support of the Ministry of Education, Youth and Sports to the HiLASE (CZ.1.05/2.1.00/
01.0027) and DPSSLasers (CZ.1.07/2.3.00/20.0143) projects, co-financed from the European Regional DevelopmentFund, this research has been supported by the grant RVO 68407700; financial support by the 'Deutsche Forschungsgemeinschaft' within the Sonderforschungsbereich 755 'Nanoscale Photonic Imaging' and the Czech Science Foundation (14-29772S) and from EU COST MPO1203, from the Ministry of Education, Youth and Sports, COST CZ VES14 LD14032 are also appreciated.

\section{References}

1. C. Phipps, Laser Ablation and its Applications (Springer, New York, 2006).

2. D. Bäuerle, Laser Processing and Chemistry 3rd edn (Springer-Verlag, Berlin, 2000).

3. T. Lippert, Plasma Process. Polym. 2, 525 (2005).

4. M. Lapczyna and M. Stuke, Appl. Phys. A 66, 473 (1998).

5. D. Riedel and M. Claude Castex, Appl. Phys. A 69, 375 (1999).

6. B. R. Benware, C. D. Macchietto, C. H. Moreno, and J. J. Rocca, Phys. Rev. Lett. 81, 5804 (1998).

7. S. Kranzusch and K. Mann, Opt. Commun. 200, 223 (2001).

8. A. Bartnik, H. Fiedorowicz, R. Jarocki, L. Juha, J. Kostecki, R. Rakowski, and M. Szczurek, Microelectron. Engng 78-79, 452 (2005).

9. L. Juha, A. Andrejczuk, R. Sobierajski, L. Ryc, F. P. Boody, H. Fiedorowicz, J. Feldhaus, V. Létal, J. Krzywinski, M. E. Grisham, P. Kubát, L. Pína, M. Horváth, G. O. Vaschenko, R. Nietubyc, Z. Otcenasek, A. Bartnik, M. Bittner, D. Chvostová, J. Mikolajczyk, J. B. Pelka, C. S. Menoni, J. J. Rocca, R. Rakowski, J. Krása, M. Kozlová, M. Pfeifer, J. Polan, A. R. Präg, B. Rus, and M. Stupka, J. Micro/Nanolith. MEMS MOEMS 4, 033007 (2005).

10. J. Chalupský, L. Juha, V. Hájková, J. Cihelka, L. Vyvsín, J. Gautier, J. Hajdu, S. P. Hau-Riege, M. Jurek, J. Krzywinski, R. A. London, E. Papalazarou, J. B. Pelka, G. Rey, S. Sebban, R. Sobierajski, N. Stojanovic, K. Tiedtke, S. Toleikis, T. Tschentscher, C. Valentin, H. Wabnitz, and P. Zeitoun, Opt. Express 17, 208 (2009).

11. http://www.sigmaaldrich.com/catalog/product/aldrich/44096 5? lang=en\&region $=\mathrm{CZ}$.

12. M. J. Bowden and J. H. O’Donnell, Dev. Polym. Degrad. 6, 21 (1985).

13. B. W. Yates and D. M. Shinozaki, J. Mater. Res. 7, 520 (1992).

14. F. Iacona and G. Marletta, Nucl. Instrum. Meth. Phys. Res. B 166-7, 676 (2000).

15. S. Heinbuch, M. Grisham, D. Martz, and J. J. Rocca, Opt. Express 13, 4050 (2005).

16. L. Vyvsín, T. Burian, J. Chalupský, M. Grisham, V. Hájková, S. Heinbuch, K. Jakubczak, D. Martz, T. Mocek, P. Pira, J. Polan, J. J. Rocca, B. Rus, J. Sobota, and L. Juha, Proc. SPIE 7361, 736100 (2009).

17. F. Barkusky, C. Peth, A. Bayer, and K. Mann, J. Appl. Phys. 101, 124908 (2007).

18. C. Liberatore, K. Mann, M. Müller, L. Pina, L. Juha, L. Vyšín, J. J. Rocca, A. Endo, and T. Mocek, Phys. Scr. T 161, 014066 (2014).

19. http://henke.lbl.gov/optical_constants/filter2.html. 